\title{
Didáctica de las matemáticas y reproducibilidad
}

\section{Mathematics Education and reproducibility}

\author{
Michèle Artigue ${ }^{1,2}$
}

Resumen: Las cuestiones de la reproducibilidad están en el corazón de los debates en torno al estatus de diversos campos de investigación y la didáctica de las matemáticas, en su ambición de constituirse como campo científico, no puede eclipsar estas cuestiones. En este artículo, considero estas cuestiones revisando un trabajo de investigación sobre la reproducibilidad de las situaciones didácticas, realizado hace treinta años, en el que se abordó esta cuestión de manera original, pero que ha permanecido aislado dentro de la comunidad didáctica.

Palabras clave: didáctica de las matemáticas, reproducibilidad, situaciones didácticas.

Abstract: The issues of reproducibility are at the heart of debates about the status of several fields of research and the didactics of mathematics, in its ambition to become a scientific field, cannot hide these issues. In this article, I address these issues by revisiting a research study on the reproducibility of

Fecha de recepción: 19 de marzo de 2018. Fecha de aceptación: 19 de abril de 2018

1 Michèle Artigue, LDAR (EA4437), Université Paris Diderot-Paris 7, UA, UCP, UPEC, URN

2 Agradezco a Avenilde Romo por hacer la traducción de este artículo originalmente escrito en francés. 
didactical situations that was developed thirty years ago, approaching this issue in an original way, but that has remained an isolated work within the didactics community.

Key words: didactics of mathematics, reproducibility, didactic situations.

\section{INTRODUCCIÓN}

Las cuestiones de reproducibilidad están en el corazón de los debates en torno al estatus de diversos campos de investigación. Popper (1959), recurrentemente citado, hace de la reproducibilidad, una de las condiciones de la cientificidad de un campo de conocimiento. Aunque se reconoce que algunos campos científicos bien establecidos, como las ciencias de la naturaleza y la astrofísica por ejemplo o algunas áreas de las ciencias de la tierra como la vulcanología, no permiten las experimentaciones controladas, generalmente asociadas a la idea de reproducibilidad en ciencias. El hecho es que cada campo científico se posiciona a través de los tipos de fenómenos, invariantes, regularidades, que aspira a identificar, y los métodos que le permiten producirlos y validarlos.

No es posible no posicionarse en relación a las cuestiones de producción y de reproducción, de situarse en relación a una visión de la ciencia como fenómeno-técnica, retomando la terminología de Bachelard (1937). La didáctica de las matemáticas no puede escapar a estos cuestionamientos. Más aún, porque está bajo presiones constantes que aspiran someterla a los modos de producción y de validación de conocimientos de las ciencias experimentales, como lo demuestra el auge del concepto de Evidence-Based Education inspirado en Evidence-Based Medecine (Biesta 2010, Wiseman 2010), y porque se espera, cada vez más, que esta disciplina pruebe su capacidad para producir, de manera controlada, efectos didácticos sobre la base de los conocimientos que ha acumulado.

Sin embargo, en la literatura de la didáctica de las matemáticas la cuestión de la reproducibilidad poco se cuestiona y también poco se trabaja explícitamente. Este hecho es el que me impulsó al inicio de los años 80 a reflexionar en este dominio en el marco de la preparación de mi doctorado de estado. Debido a que estas cuestiones me parecen todavía insuficientemente trabajadas, después de más de treinta años me he involucrado en la revisión de esta investigación que ha permanecido aislada, considerando los conocimientos de los cuales disponemos actualmente. 
En una primera parte, comentaré esta investigación y los resultados que me ha permitido establecer. Luego, en una segunda parte, cuestionaré sobre las posibles razones de su aislamiento en el campo didáctico, y sobre la manera en la cual la cuestión de la reproducibilidad podría ser retomada y trabajada hoy en didáctica de las matemáticas, conjugando los aportes conceptuales y metodológicos de este trabajo pionero y los conocimientos didácticos y científicos construidos desde entonces.

\section{UNA INVESTIGACIÓN SOBRE LA REPRODUCIBILIDAD DE LAS SITUACIONES DIDÁCTICAS}

La investigación sobre la reproducibilidad, llevada a cabo en el marco de la preparación de mi doctorado de estado, ha sido objeto de un artículo substancial (58 páginas) publicado en la revista Recherches en Didactique des Matemátiques (Artigue, 1986) y la tesis en sí, sustentada en 1984, se encuentra disponible en línea (Artigue, 1984). Parte de la conclusión obtenida en aquella época:

Cuando se busca delimitar, a través de las publicaciones, el estatus de la reproducibilidad en didáctica, uno se percata rápidamente de que su estatus rara vez es explícito y que cuando lo es, es bajo una forma esencialmente ideológica. (Artigue, 1986, p. 7 , traducción del original)

Además, según el tipo de investigaciones realizadas, se trata de regularidades de naturaleza muy distinta. La revisión que hice en aquella época de los artículos publicados en la revista Recherches en Didactique des Mathématiques, muestra que se habla de validez, de alcance de los resultados obtenidos, por ejemplo, sobre las concepciones de los estudiantes, y que los autores señalan regularmente el peligro de generalizaciones abusivas, pero las palabras reproducibilidad o sus sinónimos como repetibilidad son poco utilizadas. Cuando aparecen, es en relación a una reflexión sobre la posibilidad, las condiciones de una experimentación científica en clase como lo ilustra esta cita de Brousseau (1980), que constituye una de las raras excepciones: "A través de este estudio, surgen otras cuestiones más generales como por ejemplo ésta de la reproducibilidad en didáctica, o incluso la posibilidad de realizar experiencias científicas" (p. 56, traducción original). 
Enfaticé que esta situación conduce efectivamente a difundir, conscientemente o no, visiones implícitas de la reproducibilidad de situaciones didácticas, que no son cuestionadas. En mi trabajo de investigación, intenté modelizarlas y estudiar esos modelos.

\subsection{UNA PRIMERA MODELIZACIÓN}

El proceso de modelización comienza con la identificación de un modelo calificado de 'ingenuo', en el cual las situaciones reproducibles están relacionadas con las siguientes características.

A1 - Los mismos procedimientos deben aparecer (al menos los no marginales) con jerarquías comparables:

A2 - La historia de la clase puede ser descrita por un pequeño número de órbitas. A3 - Las regularidades observadas en el nivel de procedimientos y de las órbitas resultan esencialmente de regularidades individuales. Éstas no deben ser dependientes de las acciones de reajuste o de desbloqueo repetidas por el profesor.

A4 - Las ligeras perturbaciones que no pueden dejar de producirse de una clase a la otra no deben tener tendencia a amplificarse. (Artigue, 1986, p. 12, traducción original).

Esta descripción constituye por supuesto una simplificación de la 'realidad' que pretende ser descrita, aunque actualmente está establecido que tales simplificaciones inherentes al proceso de modelización, deben ser cuestionadas, y así contribuir a la productividad científica de la modelización. Una originalidad sin ninguna duda del trabajo llevado a cabo consiste en haber seguido esta lógica de modelización hasta el extremo, matematizando el modelo y por tanto haciéndolo accesible al cálculo. En esto, diré, que seguía de alguna manera el ejemplo dado por Brousseau, que ya había comprometido a la didáctica de las matemáticas en esta vía, utilizando modelos provenientes de la teoría de juegos (ver por ejemplo la tesis de Rajoson, 1981).

Una matematización, de naturaleza probabilista, ha sido por tanto efectuada para explorar las consecuencias posibles de tales características. En un primer tiempo, las interacciones no fueron tomadas en cuenta. El modelo fue definido para una población P (correspondiente a los estudiantes) cuyas características cognitivas son traducidas por un campo ponderado $C$, de hecho un conjunto 
finito de órbitas $\left\{O_{1, \ldots, O_{s}}\right\}$ (siendo cada órbita una serie finita de estados del sujeto y por tanto un reagrupamiento de trayectorias posibles) y una distribución de frecuencias $Q=\left\{q_{1}, \ldots, q_{s}\right\}$ sobre este conjunto. Una historia de clase con N estudiantes en un campo es entonces definida como una supla de enteros $H=\left(n_{1}, \ldots\right.$, $\left.n_{s}\right), n_{j}$ siendo el número de estudiantes en la órbita $O_{j}$, tal que la suma de las $n_{i}$ sea igual a $N$. Puesto que no se toman en cuenta las interacciones en este primer modelo, es natural asociarle a $(C, Q)$ una ley de probabilidades multinomial con $s$ parámetros $\left(N, q_{1}, \ldots, q_{s}\right)$, para la cual la probabilidad de una historia $\left(n_{1}, \ldots, n_{s}\right)$ es, con las notaciones utilizadas en el artículo igual a:

$$
\hat{Q}\left(\left\{\left(n_{1}, \ldots, n_{s}\right)\right\}\right)=\frac{N !}{n_{1} ! \ldots n_{s} !} \prod_{i=1}^{i=s} q_{i}{ }^{n_{i}}
$$

Asimismo, para considerar el hecho de que no se trata de obtener una reproducibilidad exacta, una noción de vecindad de la historia fue introducida en el modelo, la cual debía satisfacer las condiciones siguientes

V1 - La noción de vecindad debe respetar toda jerarquía marcada entre las órbitas.

V2 - Si una órbita no es marginal en una historia, ésta debe aparecer en todas las historias vecinas.

V3 - La noción de vecindad debe ser estable por reagrupamiento de órbitas. (ibídem, p. 14, traducción original).

Para los valores de s y $N$ considerados en el estudio, s entre 2 y 5 y $N$ entre 20 y 30, estas condiciones aparecen satisfechas para una distancia asociada a la suma de las diferencias absolutas y un umbral del orden de $\sqrt{ } \mathrm{N}$. Las probabilidades máximas de la historia de clase en un campo ponderado dado, que corresponden a las historias naturalmente asociadas al campo, son en todos los casos muy pequeñas, y éstas lo son máxime que la ponderación del campo es equilibrada o el número de órbitas es más grande. La introducción de vecindades aumenta estas probabilidades, pero los cálculos muestran que, para los campos que tienen dos o tres órbitas, la reproducibilidad no está asegurada en el umbral clásico de 0.1 sino para los campos que admiten una órbita fuertemente dominante. Por ejemplo, para $N=30$ y $s=2$ o 3 , con un umbral de distancia de 6 , es necesario que la ponderación de una de las órbitas sea 
superior o igual a 0.8. Y, por tanto, ieste modelo no considera la condición A4 de estabilidad en relación a las pequeñas perturbaciones del campo!

Lo que muestra claramente esta primera modelización, como lo señalamos en el artículo, es que el tamaño de la muestra de la clase es demasiado pequeño para permitir el paso de las regularidades individuales al nivel colectivo, sin procesos de interacción, y esto mismo satisfaciéndose de una visión aproximada de la reproducibilidad, como la relacionada a la noción de vecindad de la historia. La visión ingenua de la reproducibilidad que habíamos intentado modelar no parece apta sino para un caso de figura, iéste donde la cuasi-totalidad de los alumnos sigue la misma órbita!

Esta conclusión nos llevó en el artículo a generar nuevas cuestiones: ¿Será que la inadecuación del modelo ingenuo tiene como causa principal la ausencia de la consideración de los procesos de interacción al interior de la clase que, de hecho, son algunas de las condiciones esenciales de la solidez de las situaciones didácticas y de su posible reproducibilidad? ¿Será que la inadecuación del modelo ingenuo es más profunda, induciéndonos a buscar regularidades en niveles donde no pueden existir? Para intentar responderlas, voy a volver sobre una investigación llevada a cabo algunos años antes, en colaboración con Jacqueline Robinet, la cual tenía que ver con las concepciones del círculo desarrolladas en la escuela elemental, que nos permitió recoger datos substanciales, así como desarrollar ciertas situaciones, que luego fueron reutilizadas en otros contextos, y que parecían en aquella época situaciones con un fuerte potencial a-didáctico, en el sentido dado a este término en la teoría de situaciones didácticas (Brousseau, 1997), lo que hacía su dinámica menos dependiente de las acciones repetidas del profesor, y por lo tanto eran candidatas propicias para la reproducibilidad. Voy a elegir una de ellas, llamada la situación de los discos. La metodología utilizada está en consonancia con el trabajo de modelización anteriormente realizado:

- en un primer tiempo, volver sobre los datos obtenidos en las experimentaciones de esta situación y retomar los análisis ya efectuados, para intentar construir un modelo cualitativo que supere el modelo ingenuo;

- en un segundo tiempo, aprovechar el modelo construido asociándole una familia de modelos cuantitativos y estudiando las dinámicas asociadas, principalmente por medio de simulaciones informáticas;

- en un tercer tiempo, interpretar los resultados obtenidos en términos de reproducibilidad. 
Justifico esta elección en el artículo (p. 20, traducción del original):

La multiplicación de experimentaciones en la clase, sobre una situación o un proceso dado, suficientemente precisas para permitir seleccionar, de manera justificada, entre los hechos que serán fácilmente reproducibles y los que no, no es compatible con la economía de la investigación. Por lo tanto, resulta esencial encontrar medios de rentabilizar y de economizar las experimentaciones en la clase.

En esta investigación que aspiraba a la economía y a la rentabilidad, se recurrió a simulaciones informáticas. Regresaré sobre este punto en la segunda parte del artículo.

\subsection{REGRESO SOBRE LA SITUACIÓN DE LOS DISCOS}

La situación de los discos es la primera de una serie de tres. En ella los alumnos organizados en equipos de cuatro, reciben un sobre que contiene 13 sectores recortados en tres discos de cartón fino de radio diferente, los sectores de un mismo disco son de tamaño diferente. Los alumnos deben reconstruir los discos. Uno está incompleto, lo que los obliga a construir el sector faltante. Cada alumno debe construir su propio pedazo. La utilización del compás no está permitida y para evitar un tratamiento del tipo rompecabezas (puzzle), el sector faltante es más grande que los otros y no es reunión de los otros sectores.

Los datos utilizados han sido obtenidos en dos clases de CE2 (alumnos de 8 o 9 años, del tercer año de la escuela primaria) con un observador por equipo. Se identifica la emergencia de cuatro procedimientos de construcción nombrados I, R, C y P:

- I consiste en reconstruir el disco incompleto, colocarlo sobre el cartón dado para trazar el sector faltante, trazar los dos radios que lo delimitan, luego colocar entre esos dos radios el más grande de los sectores, seguir con el lápiz su borde curvo para enseguida terminarlo a mano alzada.

- $R$ consiste en construir el sector faltante haciendo rotar uno de los sectores del disco alrededor de su centro y siguiendo su contorno.

- C consiste en yuxtaponer dos sectores para obtener un sector más grande que el que debe construirse y reducirlo enseguida a la dimensión correcta. 
- P consiste en hacer un trazado punto por punto del borde curvo del sector faltante, trasladando la longitud del borde derecho de uno de los sectores a partir de su centro.

Como lo señalo en el artículo, si se lee el análisis de esta situación propuesto en (Artigue y Robinet, 1982) y es también el caso en los otros textos asociados a esta investigación, se puede decir que la visión que se impone es la de dos historias de clase que, a pesar de inevitables diferencias, obedecen básicamente a la misma dinámica: la transición de un estado inicial que corresponde a la utilización, por casi todos los estudiantes (38/40), de una misma estrategia de base, en el sentido de la teoría de situaciones didácticas -el procedimiento I que no les permite tener éxito- a un estado final donde domina fuertemente la construcción exitosa utilizando el procedimiento $R$. Los análisis realizados tornan inteligible está dinámica, contribuyendo a hacerla una dinámica previsible, y teniendo en cuenta las características de la situación y de los alumnos, y a considerar que se trata de una situación reproducible, conforme al modelo implícito descrito más arriba.

El estudio realizado de este modelo va a permitirme volver sobre los datos de esta investigación para cuestionar esta visión. Sin entrar en los detalles, este regreso va a poner en evidencia diferencias sensibles entre las dinámicas de las dos clases y también, en los dos casos, el rol esencial de las interacciones alumnos-alumnos o profesor-alumnos en dichas dinámicas. En una de las clases, en efecto, si todos los alumnos se sitúan sobre la órbita $I-R$ es porque un alumno juega un rol determinante. Él pasa de la Ry este cambio de procedimiento es, para él, muy exitoso. Él se presume en voz alta y el procedimiento $R$ difunde, primero localmente en su grupo y luego globalmente. En el otro caso, se observa al contrario la existencia de 6 órbitas diferentes: $R, P, I-C, I-P, I-E-P, I-E-P-R$ (E designa un estado de abandono momentáneo aquí), y la mayoría de los alumnos se sitúan en la última de estas órbitas. El funcionamiento no es aislado: $P$ difunde localmente para producir $I-P$; tampoco es cuasi-aislado (es decir sin intervención directa del profesor). Para las dos últimas órbitas, de hecho, el profesor interviene, percibiendo el estado de abandono de un número importante de alumnos, organiza una síntesis colectiva. En el transcurso de la síntesis, explicitando las razones del fracaso de I, el profesor da un sentido al procedimiento $P$, que de hecho es el más escolar, y favorece su difusión, aunque en ese momento este procedimiento no se hace realmente efectivo. Finalmente, si el procedimiento $R$ se impone, es como un perfeccionamiento técnico del procedimiento puntual $P$, que es de hecho el más costoso. Estos elementos serán la base de la modelización de la situación que describo a continuación y de su cuantificación. 


\subsection{MODELIZACIÓN Y SIMULACIONES}

En esta modelización, los cuatro procedimientos identificados pueden ser organizados en dos jerarquías, una jerarquía de eficacia y una jerarquía de accesibilidad. Para la primera, $C$ y $R$, son más fáciles de ejecutar correctamente y menos costosos, por lo que sobrepasan claramente Py por supuesto I, que no permite tener un resultado favorable. Para la segunda jerarquía, I se despega claramente, pero en vista de los datos obtenidos, es más difícil jerarquizar los otros tres. Sin embargo, sabiendo que $P$ moviliza una concepción puntual del círculo, parece, en vista de los conocimientos adquiridos en la investigación, menos accesible que los otros dos. Considerando $C$ y $R$, la jerarquía es menos clara, y la segunda situación, la de las coronas, donde el estado final en las dos clases corresponde a la adopción masiva de $C$, tiende a confirmarlo. La intervención de estas dos jerarquías permite inscribir las situaciones observadas en un contexto más amplio presentando las características siguientes:

- existe una inversión clara de las jerarquías entre eficacia y accesibilidad, lo que al mismo tiempo convierte a la situación en no trivial;

- el medio (milieu) ofrece una retroalimentación eficaz y rápida para la validación/invalidación de los procedimientos (aquí, efectivamente, la retroalimentación perceptiva es particularmente eficaz, como lo es en la situación del rompecabezas de Brousseau, una situación clave de su ingeniería sobre números racionales y decimales. (Brousseau, Brousseau y Warfield 2014);

- la rapidez de ejecución del procedimiento más accesible y la distancia con los otros procedimientos, es lo que hace que los estados de bloqueo sean más susceptibles de aparecer;

- la facilidad con la cual la validez de un procedimiento puede ser verificada (aquí perceptivamente) y dicho procedimiento se puede difundir, al menos localmente (aquí en vista de las acciones asociadas);

- pero también, es la dificultad de la tarea, lo que disminuye el número de posibles centros de difusión.

Es sobre la base de estas características que se elaboró un modelo cualitativo que toma la forma de un grafo $G$ ponderado, donde los vértices representan los estados posibles (estado de inicio, estado de ejecución de procedimientos, estados finales de éxito, estado de abandono) y las aristas los posibles cambios de estado. Estas aristas estarán asignadas a probabilidades de transición luego 
de la cuantificación del modelo. En este modelo, el tiempo está gestionado por un contador discreto, cada procedimiento $Q_{i}$ está asignado a un tiempo de ejecución $t_{i j}$ y a una tasa de repeticiones permitidas $m_{i}$. El grafo $G$ correspondiente (esquema 2 en el artículo), sin ponderaciones, es reproducido en la figura 1, donde $A C, A R$ y $A P$ representan un estado final de éxito en relación con los procedimientos $C, R$ y $P$, y E el estado de abandono.

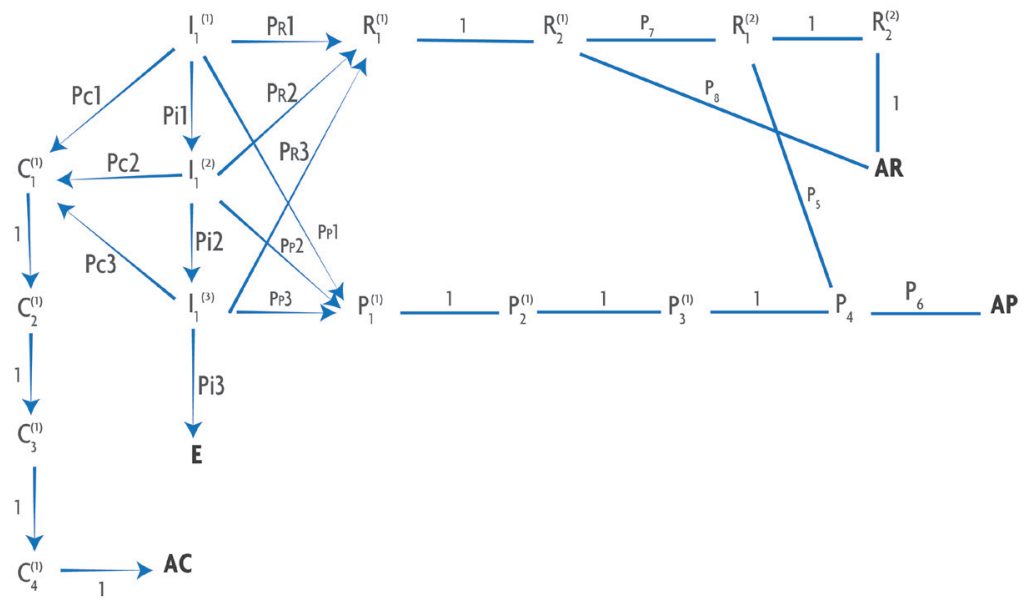

Figura 1. "Grafo G"

En cuanto a las interacciones, éstas son modelizadas por un sistema de reglas de difusión cuya acción se superpone a ésta del grafo. Se distingue entre una difusión local al nivel de los equipos y una difusión más global que, también serán ponderadas luego de la cuantificación del modelo.

Es posible apreciar que, a pesar de que en esta modelización intervienen un gran número de parámetros, no es sino una drástica simplificación de la realidad de la clase. Por otra parte, los datos obtenidos permiten considerar solamente intervalos de valor razonable para estos parámetros. La modelización conduce por lo tanto a una infinidad de modelos cuantitativos que en la investigación exploran. Esta exploración privilegia tres variables consideradas clave por la dinámica de la situación en vista de las observaciones realizadas: la probabilidad de abandono, el tamaño de los equipos y el coeficiente de difusión local. De hecho, 16 cuantificaciones del modelo van a ser exploradas correspondientes a dos probabilidades de abandono (vecinas de 0.5 y 0.7), dos tamaños de equipos (2 y 4 estudiantes) y 4 
valores del coeficiente de difusión global $(0,0.25,0.5$ y 1$)$. El grafo ponderado asociado es el grafo $G^{\prime}$ que se reproduce a continuación (ver figura 2), en el cual las aristas tienen las ponderaciones de la tabla 1. Este grafo es una versión simplificada del grafo $G$, los tiempos de ejecución y la tasa de repetición admitida para cada procedimiento son fijos (por ejemplo, de valor 1 y 4 respectivamente para el procedimiento I) así como las probabilidades de transición entre procedimientos.

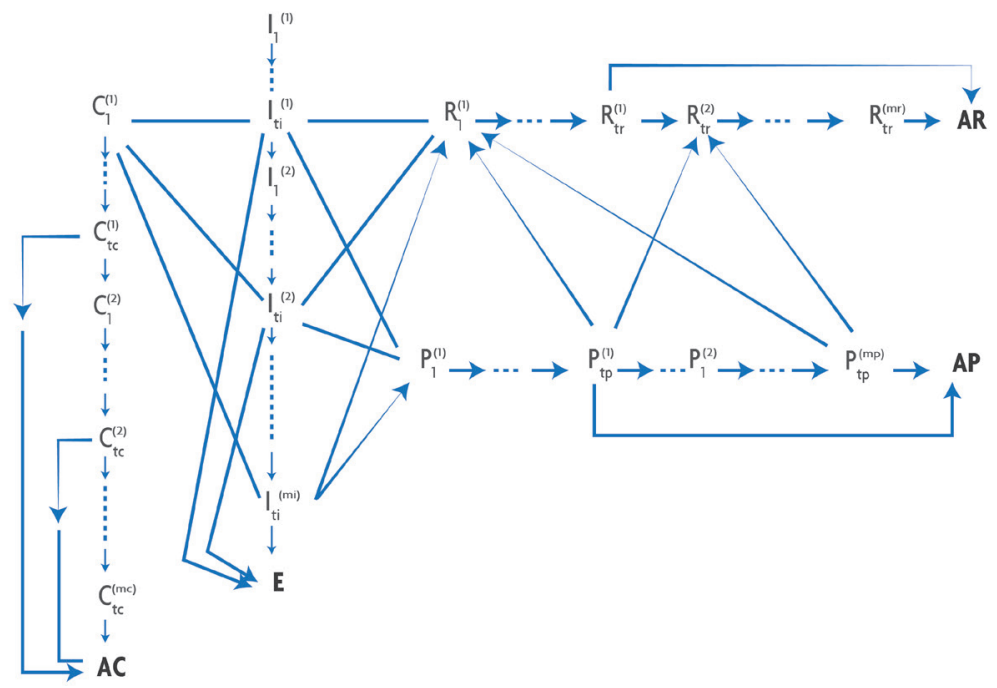

Figura 2. "Grafo G'"

Tabla 1. Ponderaciones asociadas al grafo G

\begin{tabular}{|c|c|c|c|c|c|c|c|c|c|c|c|c|}
\hline PONDERACIONES & Pi1 & Pi2 & Pi3 & Pr1 & Pr2 & Pr3 & Pc1 & Pc2 & Pc3 & Pp1 & Pp2 & Pp3 \\
\hline$(1)$ & .9 & .8 & .7 & .04 & .08 & .12 & .04 & .08 & .12 & .02 & .04 & .06 \\
\hline$(2)$ & .95 & .9 & .82 & .02 & .04 & .07 & .02 & .04 & .07 & .07 & .02 & .04 \\
\hline
\end{tabular}

Para los modelos sin difusión o con difusión local por equipos de 2, los cálculos relativamente simples de probabilidades han permitido determinar los estados finales de clase y las probabilidades en términos de vecindades, así como la duración de las historias de clase. Pero, para el estudio de otros modelos, esto resultaba muy complejo, por lo que se recurrió a simulaciones informáticas 
utilizando un método de Montecarlo para simular las transiciones de estado. El organigrama del programa de simulación aparece en el anexo. Para cada caso, se han realizado 2 series de 100 simulaciones de historias de clase con 20 alumnos, no obstante, se han hecho paralelamente los cálculos para el modelo sin difusión asociada, Ilamado aquí de la historia simple asociada. Finalmente, para cada simulación, el programa provee:

- la sucesión de estados de clase, alumno por alumno;

- el estado final de la clase bajo forma de cuádruple (NE, NR, NC, NP) asociado al número de alumnos en los estados respectivos E, AR, AC y AP;

- la duración de la historia;

- el estado final de la clase para la historia simple asociada;

- la duración de esta historia simple;

- la distancia del estado final de esta historia simple simulada al estado final de la historia simple calculada teóricamente, utilizada como medio de control de la simulación.

Para cada serie de 100 simulaciones, el programa provee igualmente:

- las medias y distribuciones de los estados finales de clase, de las duraciones de la historia y de las duraciones de bloqueo;

- los mismos datos para las historias simples asociadas;

- las medias y distribuciones de las distancias de las historias simples simuladas al estado final calculado teóricamente.

Sin entrar en los detalles de estos cálculos, ni del análisis efectuado sobre los datos de las simulaciones, enviando para ello al lector a la tesis o al artículo citado, me limito aquí a sintetizar los resultados principales. A pesar del número limitado de simulaciones efectuadas para cada modelo cuantificado, aparecen fuertes regularidades.

Las simulaciones muestran, por ejemplo, que la difusión, desde que es considerada, reduce substancialmente las probabilidades de abandono. Una difusión simplemente local, por equipo de dos, es suficiente, en promedio, para compensar la diferencia entre las dos ponderaciones contempladas para la transición hacia el estado $E$ en las simulaciones. Por otra parte, las diferencias importantes que fueron observadas, según el tamaño de los equipos, en caso de difusión local, disminuyen desde que la difusión deja de ser simplemente local. 
Tratándose de los estados finales y de su distribución, la simulación de las historias simples muestra que sus estados finales siguen una ley multinomial con cuatro parámetros, y cada uno de sus componentes una ley binomial. Pero con la introducción de la difusión, las distribuciones se alejan sensiblemente de este modelo, la diferencia aumenta cuando el coeficiente de difusión crece, como podía esperarse. En todos los casos, las diferencias observadas son estadísticamente significativas. Las regularidades observadas en este nivel me llevaron a introducir la noción de historia con estado dominante, definida como una historia en la cual uno de los componentes del estado final reagrupa más del 70\% de los alumnos. Desde que la difusión ya no es simplemente local, estas historias se convierten en mayoría, como lo muestra la tabla 2 que aparece a continuación y que corresponde a la tabla 10 del artículo. En esta tabla, arriba de la diagonal aparecen los porcentajes globales y abajo los porcentajes de historias con un estado final dominante AR. El asterisco indica la existencia de historias con un estado final $E$ dominante. Como lo muestra esta tabla, y en coherencia con lo que ha sido mencionado, para los abandonos, la variable ponderación de los abandonos tiene mayor influencia que la variable tamaño de los equipos.

Tabla 2. Porcentaje de las historias de clase con un estado final dominante

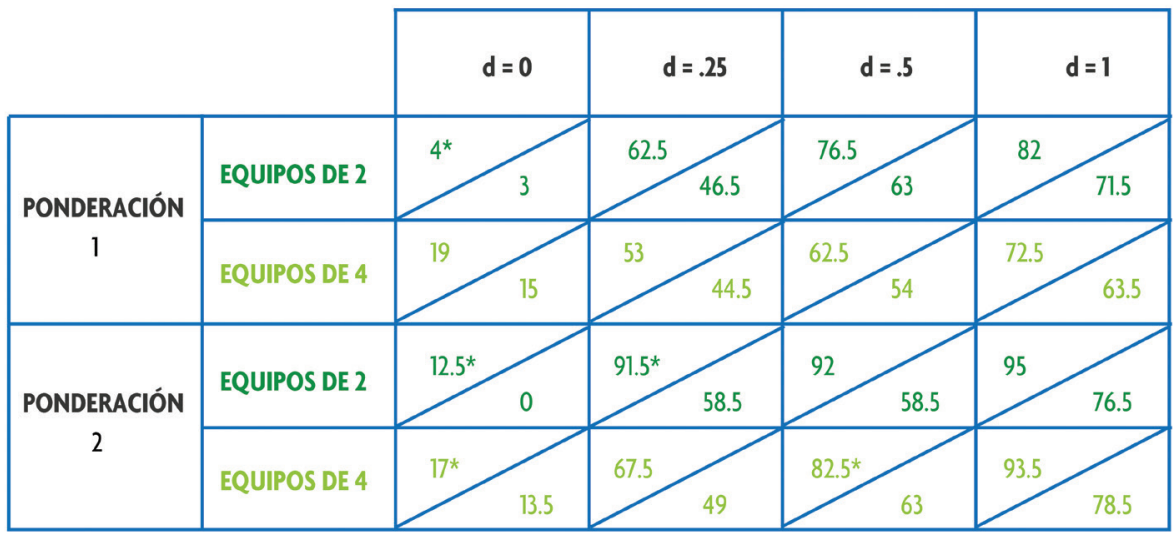

Para resumir, como se ha hecho en el artículo (p.52), lo que tiende a mostrar finalmente esta exploración de los 16 modelos cuantitativos asociados al modelo cualitativo elaborado, es: 
- la imposibilidad, dadas las características de la situación citada anteriormente, de garantizar su viabilidad en el marco de un funcionamiento sin interacción, o incluso sólo con interacción local;

- la viabilidad en revancha de una gestión de clase donde el profesor, sin aportar directamente información, facilitaría el paso de una difusión local, al interior de los equipos, a una difusión más global, al interior de la clase;

- la alta probabilidad de que, en el marco de un tal funcionamiento, aparezca un estado final de clase dominante, en un tiempo compatible con las exigencias del sistema escolar, pero no parece posible garantizar que se tratará del estado final AR, observado en las dos clases.

\section{CONCLUSIONES}

El artículo se termina con una larga conclusión donde, apoyándome en el estudio realizado, vuelvo a las cuestiones iniciales relativas a la reproducibilidad de las situaciones didácticas, y principalmente a la de investigar el nivel de las regularidades. Para la situación de los discos, en vista de los resultados mencionados anteriormente, explico que me parece inútil querer investigar la reproducibilidad al nivel de las historias de clase, pero que las características de la situación permiten esperar una reproducibilidad a nivel de estructura de las historias, una reproducibilidad "estructural", en cuya producción sin ninguna duda el maestro desempeñará un rol decisivo, ya que sólo permitiendo la difusión de los procedimientos ganadores más allá del nivel puramente local. Vuelvo también a la conclusión sobre las particularidades de esta situación y de su gestión. Para el lector que no está familiarizado con la teoría de las situaciones didácticas, preciso un poco más lo que en el artículo es más alusivo. No se trata de una sesión de clase ordinaria. La sesión está diseñada con fines de investigación. Se trata de estudiar las concepciones del círculo puestas en marcha por los estudiantes en la resolución de una tarea específica, que permite movilizar a priori varias concepciones, no solamente la del círculo como un conjunto de puntos a una distancia dada de un punto fijo dado, sino también, por ejemplo, la concepción del círculo como curva plana cerrada de curvatura constante. Además, la serie de situaciones, borrando progresivamente el centro del círculo (sectores de discos - pedazos de coronas circulares- arcos de círculos) aspira a favorecer cada vez más esta concepción. Las diferentes situaciones están diseñadas en referencia a la teoría de situaciones didácticas, como situa- 
ciones de acción (Brousseau, 1997). Las variables didácticas han sido cuidadosamente elegidas, al igual que las características del medio (milieu) con el cual interactúan los alumnos. Estas interacciones con el medio (milieu), diseñado como medio antagonista en el sentido de la teoría, deben permitir invalidar la estrategia de base, pero también posibilitar la movilización de otras estrategias, si las concepciones asociadas están disponibles en los estudiantes. El funcionamiento inicialmente previsto es un funcionamiento, cuasi-aislado del profesor, en el sentido definido anteriormente, permitido por el potencial a-didáctico de la situación. Este tipo de situación se diseña para devolver a los alumnos la responsabilidad matemática de la resolución de las tareas propuestas, pero como se ve aquí, cuando las interacciones con el medio (milieu) no resultan suficientemente productivas, el profesor debe intervenir. Es en relación a esta constatación que en el artículo surge la distinción entre dos formas de reproducibilidad: una externa al nivel de historias de clase y otra al nivel del sentido, la cual abordaré más ampliamente en la siguiente parte:

En la ausencia de informaciones precisas tanto sobre certidumbres como incertidumbres de su dinámica [la de las situaciones], sobre los momentos claves de decisión, el profesor puede tener tendencia a forzar la reproducción de dinámicas ya observadas o descritas en los documentos, la realización de esta reproducibilidad externa haciéndose frecuentemente contra una reproducibilidad al nivel del sentido. (ibídem, p. 56, traducción del original).

Finalmente, cuestionando el alcance de este estudio, vuelvo sobre las características de la situación estudiada y sobre la metodología de la investigación. En cuanto a las características, las detallo nuevamente en una formulación descontextualizada, enfatizando que, si no son las de las situaciones habituales de enseñanza, tampoco son propias a esta situación particular y que por lo tanto "este estudio debería permitir avanzar en el estudio de los problemas relacionados con la reproducibilidad no solamente de esta situación específica sino de toda una clase de situaciones" (ibídem, p. 57, traducción del original). En cuanto a la metodología de la investigación, vuelvo en particular al uso de simulaciones informáticas:

El método utilizado para la exploración de un modelo teórico a través de simulaciones en la computadora ha permitido ampliar nuestro campo de visión, liberándonos de realizaciones específicas observadas. Además, nos ha dado elementos para comprender de mejor manera la influencia de las diferentes variables didácticas en la 
historia de la clase. Esto debe permitir interpretar mejor y más rápidamente la evidencia que se podría recopilar, en tiempo real, en las observaciones posteriores y en última instancia dar al profesor una manera más efectiva de actuar, para mantener la viabilidad del sistema, dejando, sin embargo, a los alumnos la responsabilidad de la investigación. (ibídem, p. 57, traducción del original).

Al término de esta investigación, el costo razonable de estas simulaciones informáticas, la inteligibilidad que han aportado en el estudio realizado, me conducen a pensar que esta metodología puede ser prometedora, para guiar experimentaciones de clase que no se pueden multiplicar y optimizar el provecho que es posible obtener de éstas.

\section{REFLEXIONES Y PERSPECTIVAS}

En aquella época la metodología me parecía prometedora, sin embargo, ésta no se ha vuelto a utilizar, ni por mí ni por otros investigadores y esta investigación que Hans Freudenthal, sinodal de esta tesis de estado, había reconocido por su originalidad y calificado como el primer trabajo matemático de la didáctica, ha quedado como una obra aislada. Personalmente, mis intereses se orientaron hacia la didáctica del análisis en la universidad. En efecto, por una parte, las secciones experimentales físico-matemáticas que creamos en la Universidad París 7, en esa época, ponían de manifiesto los problemas asociados a la enseñanza de los diferenciales y me comprometí en un trabajo co-disciplinar en este tema (Alibert et al., 1988), por otra parte, mis intereses matemáticos cambiaron de la lógica hacia los sistemas dinámicos. Después de haber publicado con mi colega Véronique Gautheron un libro sobre el estudio cualitativo de los sistemas diferenciales autónomos (Artigue y Gautheron, 1983), me involucré en una investigación didáctica dirigida a estudiar la posibilidad de implementar en un primer año universitario, un curso de ecuaciones diferenciales más conforme con la epistemología del campo, introduciendo principalmente una aproximación cualitativa de la resolución de las ecuaciones diferenciales (Artigue y Rogalski, 1990). De hecho, no es difícil percibir en la investigación descrita anteriormente, la influencia de este interés por los sistemas dinámicos. Es en términos de las propiedades de la dinámica de un sistema complejo que se plantea la pregunta de la reproducibilidad; las nociones de órbita y de campo están en el corazón de la modelización; son las propiedades esencialmente cualitativas de la diná- 
mica de la situación de los discos las que son pretendidas, incluso si el estudio pasa por la cuantificación de los modelos; finalmente la idea de investigar los invariantes en el nivel de la estructura de las historias puede ser relacionado con el concepto de estabilidad estructural de los sistemas dinámicos.

Sin embargo, volví sobre esta cuestión de la reproducibilidad en el curso impartido en la Escuela de Verano de didáctica de las matemáticas en 1989 sobre la ingeniería didáctica, el cual también fue publicado en la revista Recherches en Didactique des Mathématiques (Artigue, 1989). Primero, establecí la relación entre reproducibilidad y obsolescencia de las situaciones didácticas. Esta noción había sido introducida por Brousseau (1981), quien se enfrentaba al problema de la reproducción, año tras año, en la Escuela Michelet del COREM (Centro de Observación para la Investigación sobre la Enseñanza de las Matemáticas), de la ingeniería didáctica que él había diseñado para la enseñanza de los números racionales y decimales (para un análisis retrospectivo particularmente interesante de esta ingeniería, envío al lector a la obra reciente (Brousseau, Brousseau y Warfield 2014). Brousseau define así la obsolescencia:

Por obsolescencia nos referimos al fenómeno siguiente: los maestros, de un año al otro, tienen cada vez más dificultades para reproducir las condiciones que pueden engendrar en los alumnos, tal vez a través de reacciones diferentes, una misma comprensión de la noción enseñada. En lugar de reproducir condiciones que generan el mismo resultado dejando libres las trayectorias, reproducen, por el contrario, una "historia", un desarrollo similar al de los años anteriores, mediante intervenciones que, aunque discretas, alteran las condiciones didácticas que garantizan un significado correcto de las reacciones de los alumnos. (Brousseau, 1981, p. 85, traducción del original).

En este fenómeno de obsolesencia es claramente visible la tensión que existe entre las dos formas de reproducibilidad distinguidas en la parte precedente, una reproducibilidad externa a nivel de las historias y una reproducibilidad interna a nivel del sentido, así como la idea de que la reproducibilidad externa, si se produce, va a ser forzada por acciones del profesor, más o menos conscientes. Todo esto entraba en consonancia con los resultados de mi propia investigación y me condujo, retomando de la física la idea del principio de incertidumbre, a formular la hipótesis siguiente:

Es en términos de relación de incertidumbre que conviene considerar las relaciones entre reproducibilidad externa e interna. En otros términos, una fuerte exigencia 
de reproducilidad externa no puede ser satisfecha sino sacrificando la reproducibilidad interna (que es, de hecho, la que se pretende). (Artigue 1989, p. 301, traducción del original)

Luego, me pareció que esta hipótesis tenía respaldo en trabajos contemporáneos como los de Arsac y Mante (1989) que, mostrando el efecto macroscópico de decisiones aparentemente microscópicas sobre el funcionamiento de clase, confirman la idea de que:

[...] el objeto clase se acerca más al punto de vista dinámico de los sistemas caóticos que de los sistemas estables a los cuales se refiere implícitamente el modelo ingenuo. Pero, éstos [trabajos] muestran similarmente la dificultad que puede existir para la didáctica, el integrar estos fenómenos de control fino en la modelización, si no es, como ya lo había realizado G. Brousseau en su trabajo sobre la obsolescencia, buscando identificar indirectamente su existencia a través de un cierto número de indicios, entre los cuales debería aparecer, siempre y cuando las hipótesis hechas anteriormente estuvieran bien fundamentadas, una muy buena reproducibilidad externa. (Artigue, 1989, p. 301, traducción del original)

Una vez más, el lector notará la referencia a la teoría de los sistemas dinámicos. Añadiré que sistema caótico no quiere decir que hay ausencia de regularidades como es la idea común del caos y que numerosas divulgaciones de esta teoría favorecen, sino de regularidades ubicadas a otros niveles que los que se podrían considerar espontáneamente. Etienne Ghys lo ilustró particularmente bien en su conferencia plenaria en el congreso ICME 12 (Ghys 2015).

Hoy, releyendo esta cita, no puedo dejar de ver entre líneas, la conciencia expresada implícitamente, de la dificultad que plantea la continuación del trabajo de modelización emprendido en mi primera investigación sobre la reproducibilidad de las situaciones didácticas. Una dificultad, una incertidumbre con respecto a los conocimientos que podrían resultar, lo que probablemente explica por qué no ha sido prolongada por mí o por otros, a pesar de que había sido bien recibida dentro de la comunidad didáctica. Con la retrospectiva que los años permiten, me parece que las cuestiones de modelización, de modelización estocástica de sistemas dinámicos, del uso de simulaciones informáticas, no habían sido consideradas en el campo de las ciencias matemáticas y más ampliamente en el campo científico, como lo son ahora. Con la relectura, mi proyecto de modelización de situaciones didácticas en vista de estudiar su 
dinámica aparece como un bricolaje ingenioso, pero un objeto muy distante de lo que permitiría hoy la colaboración con especialistas de la modelización de dinámica de sistemas complejos.

No por ello deja de ser cierto que estas cuestiones de reproducibilidad de situaciones didácticas son esenciales, porque es en términos de propuestas de sesiones y de secuencias de clase que muy a menudo se expresan los vínculos entre investigación y acción didáctica, con construcciones que provienen de las ingenierías didácticas de investigación, o más generalmente de los trabajos de "design-based research". A pesar del progreso de la investigación, me parece que muchos, e incluso la mayoría de estos recursos difunden siempre, implicitamente, sin que los autores sean conscientes, un modelo ingenuo de la reproducibilidad de situaciones didácticas, centrado más sobre las historias, las trayectorias, que sobre las condiciones que las hacen posibles. Al hacerlo, incluso involuntariamente, conducen a la búsqueda de una reproducibilidad externa, en detrimento de la reproducibilidad interna que por sí sola puede garantizar los aprendizajes previstos.

Personalmente, como lo expresé durante la conferencia asociada a la recepción de la medalla Felix Klein en el congreso ICME-13 (Artigue, 2017), para la cual elegí como tema el desafío de las relaciones entre investigación fundamental y acción didáctica, esta investigación sobre la reproducibilidad de las situaciones didácticas, incluso aislada, influyó profundamente sobre mi relación con la ingeniería didáctica y con la difusión de los resultados que esta metodología de investigación permite obtener. Desde la investigación sobre la enseñanza de las ecuaciones diferenciales evocada anteriormente, como escribí:

I tried to overcome the trap of linear descriptions and to open the dynamics of situations, envisaging, for instance, possible bifurcations. I also tried to approach more explicitly the key issue of the sharing of mathematical responsibility between teacher and students than was usual in classical engineering design at that time and whose underestimation appeared as a major source of denaturation. (Artigue 2017, p. 153)

Aunque no podría afirmar que en mis escritos posteriores, logré escapar a la trampa de descripciones favoreciendo interpretaciones ingenuas de la reproducibilidad. Sin embargo, a partir de los años 90, los conocimientos didácticos provenientes de la atención dada al profesor, su desarrollo profesional y sus prácticas (Chevallard, 1999; Robert y Rogalski, 2002; Adler, Ball, Krainer, Lin y Novotna, 2005), del trabajo que se ha venido desarrollando durante los últimos diez años, en el marco de la aproximación documental de lo didáctico (Gueudet, Pepin y Trouche, 2012) nos dio 
acceso a esta dimensión esencial de la actividad del profesor, del desarrollo de concepciones más colaborativas, de las relaciones entre profesores e investigadores y del concepto relacionado de ingeniería colaborativa, así como la propia evolución científica mencionada anteriormente, nos brindan nuevos incentivos para abordar estas cuestiones y superar definitivamente las visiones ingenuas.

Al preparar este artículo, quise cuestionar, desde este punto de vista, la obra resultante del reciente estudio ICMI 22 dedicado al Task design' (Watson \& Ohtani 2015). Esta obra, de una riqueza innegable, pone en evidencia la diversidad de principios y de marcos teóricos desarrollados y/o utilizados para fundamentar y analizar el análisis del diseño de tareas y de secuencias de tareas, así como su uso, una diversidad ilustrada por el estudio de numerosos ejemplos. Hace hincapié en que el diseño continúa de alguna manera en el uso, mediante las adaptaciones, las transformaciones efectuadas por los profesores; se cuestiona sobre la manera de guiar estas adaptaciones y transformaciones, percibidas como inevitables e incluso deseables porque permiten a los profesores expresar su creatividad, para preservar las causas de aprendizaje y la epistemología que ha fundamentado el diseño inicial de las tareas, y evitar las distorsiones comúnmente observadas. Pero las cuestiones estudiadas no están relacionadas con las cuestiones de reproducibilidad. Ni el término 'reproductibility', ni términos vecinos, forman parte del índice de esta obra, y la búsqueda de su presencia en el archivo pdf de la misma no señala ninguna incidencia. Las solas incidencias de la palabra 'reproduce' se encuentran en el capítulo 8, dedicado precisamente a la ingeniería didáctica, y en mi capítulo de comentario. El término 'invariante' en sí mismo, tiene pocas incidencias y aparece estrechamente relacionado a las referencias de la teoría de variaciones como fundamente del diseño (Marton, Runesson y Tsui 2004), y por lo tanto en un contexto diferente. En la didáctica francesa, estas cuestiones de reproducibilidad han estado, de hecho, relacionadas a la investigación de situaciones robustas, para las cuales la selección de las variables didácticas, la organización del medio (milieu) permiten, bajo condiciones que es necesario precisar, una dinámica cognitiva de los alumnos y de la clase que no están subordinadas a las intervenciones reiteradas del profesor, y ofrecen una cierta resistencia a las ineludibles variaciones, de una realización a la otra. Una búsqueda de incidencias de los términos 'robust' y 'robustness' en la obra brinda dos apariciones en relación con nuestro propósito. La primera, en el capítulo 2 dedicado a los principios y marcos teóricos del diseño, hace referencia a los principios que guían el diseño en términos de recorridos de estudio y de investigación en la teoría antropológica de lo didáctico: "Piloting is an 
essential phase in the design process for checking conjectured teaching and learning processes and for improving the ecological and economic robustness of a task." (ibídem, p.45). La segunda, aparece ubicada en la conclusión del capítulo 4 dando cuenta del diseño de tareas desde el punto de vista del estudiante da continuidad a una referencia de Sarrazy y Chopin (2010) y expresa el deseo de futuras investigaciones sobres estas cuestiones:

More generally, particularly helpful might be research that addresses the question: are some tasks more robust than others? The robustness of tasks might be intended as resistants to changes from the teachers but also understandable and useful for all the students. (ibídem, p. 137)

¿Debemos ver una coincidencia fortuita en el hecho de que todas las incidencias encontradas en esta obra, de una u otra manera, están relacionadas con las investigaciones de lo que comúnmente se conoce como la escuela didáctica francesa?

No me cabe duda que las cuestiones que dieron origen a mi investigación sobre la reproducibilidad en didáctica de las matemáticas, fueron y siguen siendo cuestiones ampliamente abiertas e insuficientemente trabajadas, que hoy podrían abordarse de manera más eficaz que como lo pude hacer en aquel momento y con un mayor impacto sobre las relaciones entre investigación y acción didáctica. Tampoco me cabe duda que la investigación de las situaciones robustas, su posible caracterización a la luz de los avances de la investigación, su puesta a prueba en contextos variados, la elucidación de los efectos de estos cambios de contexto y la comprensión de sus razones, es un elemento esencial para progresar sobre estas cuestiones, y más globalmente para alcanzar relaciones más eficaces entre teoría y práctica, entre investigación fundamental y acción. Por otra parte, las relaciones de los didactas con los investigadores en Entornos informáticos de aprendizaje humano (EIAH) han conducido y conducen a los didactas a hacer algunos de sus modelos de aprendizaje "calculables" para ser implementados en la computadora, como lo muestran en Francia, Ios trabajos iniciados por Brigitte Grugeon y Elisabeth Delozanne con el programa computacional Pépite, hace ya dos década, y desarrollados desde entonces sin interrupción (Pilet, Chenevotot, Grugeon, El Kechai y Delozanne, 2013), o los desarrollados en el Laboratorio de Informática de Grenoble (ver por ejemplo Chaachoua (2010)). Quizá ha llegado el momento de cuestionarse nuevamente sobre el posible aporte de las simulaciones a la investigación en didáctica. Me atrevo a esperar que la reflexión desarrollada en este artículo pueda contribuir a ello. 


\section{REFERENCIAS}

Adler, J., Ball, D. Krainer, K., Lin, F.L., \& Novotna, J. (2005). Reflections on an emerging field: Researching mathematics teacher education. Educational Studies in Mathematics, 60(3), 359-381.

Alibert, D., Artigue, M., Courdille, J.M., Grenier, D., Hallez, M., Legrand, M., Menigaux, J., Richard, F., Viennot, L. (1988). Le thème "différentielles» - un exemple de coopération maths-physique dans la recherche. In G. Vergnaud, G. Brousseau \& M. Hulin (Eds.), Didactique des acquisitions des connaissances scientifiques. Actes du Colloque de Sèvres. Mai 1987. (pp. 7-45). Grenoble: La Pensée Sauvage éditions.

Arsac, G., \& Mante, G. (1989). Le rôle du professeur - aspects pratiques et théoriques, reproductibilité. Cahiers du Séminaire de didactique des mathématiques et de l'informatique, 79-105. Greobile: IMAG-LSD.

Artigue M. \& Robinet J. (1982). Conceptions du cercle chez des enfants de l'école élémentaire. Recherches en Didactique des Mathématiques, 3(1), 5-64.

Artigue M. \& Gautheron V. (1983). Systèmes différentiels. Etude Graphique. Paris : Cedic - Fernand Nathan.

Artigue M. (1984). Contribution à l'étude de la reproductibilité des situations didactiques. Thèse d'Etat (première partie). https://tel.archives-ouvertes.fr/tel-01250658/ document

Artigue M. (1986). Étude de la dynamique d'une situation de classe: une approche de la reproductibilité. Recherches en Didactique des Mathématiques, 7(1), 5-62.

Artigue M. (1989). Ingénierie didactique. Recherches en Didactique des Mathématiques, 9(3), 281-308.

Artigue M \& Rogalski M. (1990). Enseigner autrement les équations différentielles en DEUG. In Commission interIREM Université (Ed.), Enseigner autrement les mathématiques en DEUG A première année (pp. 113-128). Lyon: LIRDIS.

Bachelard, G. (1937). La formation de l'esprit scientifique. Contribution à une psychanalyse de la connaissance objective. Paris: J. Vrin.

Biesta, G. J.J. (2010). Why 'What works' still won't work: From evidenced-based education to value-based education. Studies in Philosophy of Education, 29, 491-503.

Brousseau, G. (1980). Problèmes de l'enseignement des décimaux. Recherches en Didactique des Mathématiques, 1(1), 11-60.

Brousseau, G. (1981). Problèmes de didactique des décimaux. Recherches en Didactique des Mathématiques, 2(1), 37-128.

Brousseau, G. (1997). Theory of Didactical Situations in Mathematics. Dordrecht: Kluwer Academic Publishers. 
Brousseau, G., Brousseau, N., \& Warfield, V. (2014). Teaching Fractions through Situations: A Fundamental Experiment. New York : Springer.

Chaachoua, H. (2010). La praxéologie comme modèle didactique pour la problématique EIAH. Etude de cas : Ia modélisation des connaissances de lélève. Habilitation à diriger les recherches. Université de Grenoble. https://tel.archives-ouvertes.fr/tel00922383/document

Chevallard, Y. (1999). L'analyse des pratiques enseignantes en théorie anthropologique du didactique. Recherches en didactique des mathématiques, 19(2), 221-266.

Ghys, E. (2015). The butterfly effect. In S.J. Cho (Ed.), The Proccedings of the 12TH International Congress on Mathematics Education (pp. 19-39). New York : Springer. DOI 10.1007/978-3-319-12688-3_6

Gueudet, G., Pepin, B., \& Trouche, L. (2012). From text to 'Lived resources': Mathematics Curriculum Material and Teacher Development. New York: Springer.

Marton, F., Runesson, U., \& Tsui, B.M. (2004). The space of learning. In F. Marton \& A.B. Tsui (Eds.), Classroom discourse and the space of learning (pp. 3-40). Mahwah, NJ: Lawrence Erlbaum.

Pilet, J., Chenevotot, F., Grugeon, B., El Kechaï, N., \& Delozanne, E. (2013). Bridging diagnosis and learning of elementary algebra using technologies. In B. Ubuz, C. Haser, $\&$ M. A. Mariotti (Eds.), Proceedings of the 8th Congress of the European Society for Research in Mathematics Education (pp. 2684-2693). Ankara: Middle East Technical University and ERME.

Popper, K. R. (1959). The logic of scientific discovery. Hutchinson, London, United Kingdom.

Robert, A., \& Rogalski, J. (2002). Le système complexe et cohérent des pratiques des enseignants de mathématiques: une double approche. Revue canadienne de l'enseignement des mathématiques, des sciences et des Technologies, 2(4), 505-528.

Sarrazy, B., \& Chopin, M.-P. (2010). Anthropo-didactical approach to teacher-pupil interactions in teaching mathematics at elementary school. Scientia in Educatione, 1(1), 73-85.

Watson, A., \& Ohtani, M. (Eds.) (2015). Task design in mathematics education. An ICMI study 22. New York: Springer.

Wiseman, A.W. (2010). The uses of evidence for educational policy making. Global contexts and international trends. Review of Research in Education, 34(1), 1-24. 
Anexo: Organigrama del programa de simulación

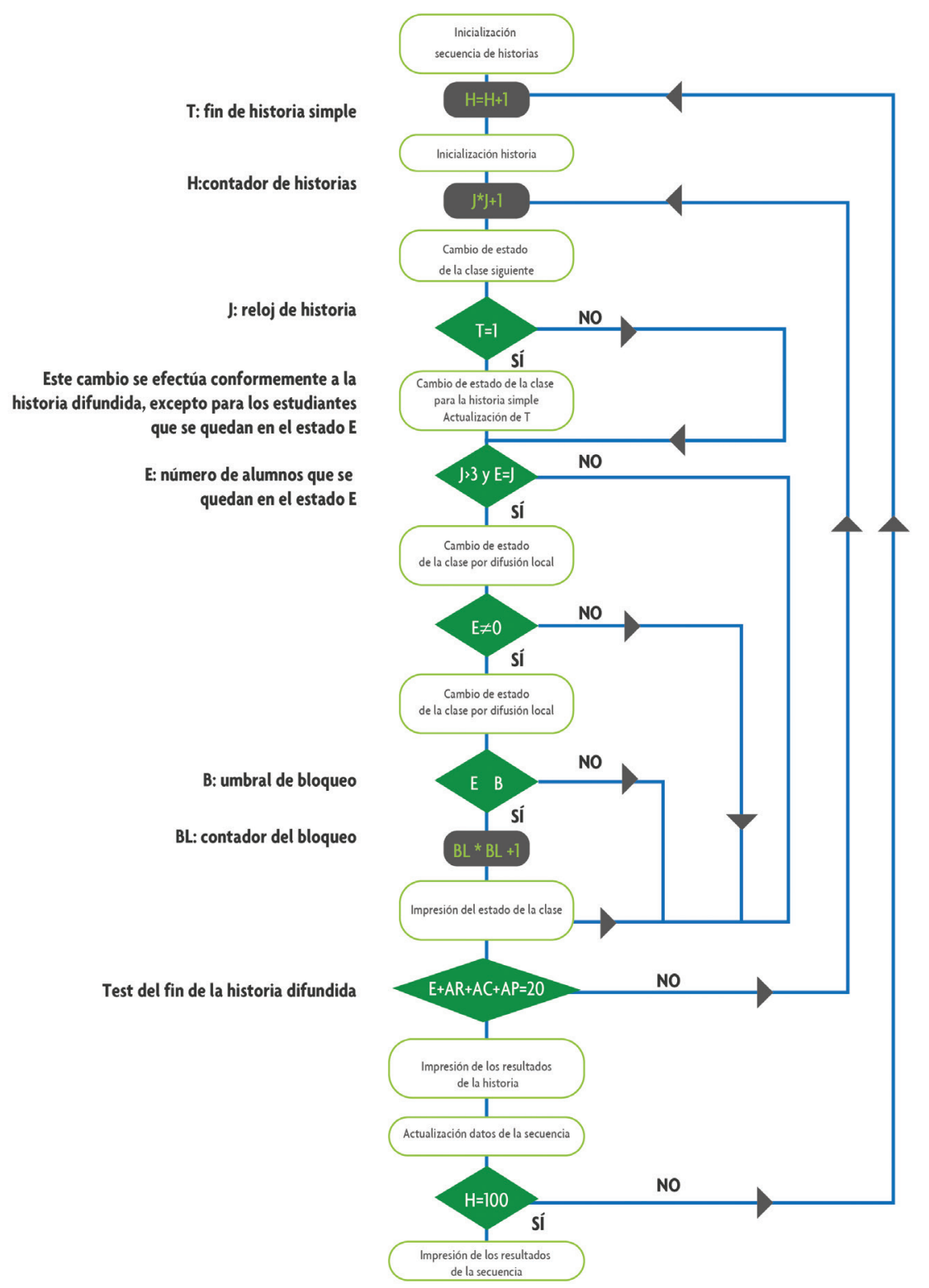

